\section{Case Reports in Nephrology and Dialysis}

Case Rep Nephrol Dial 2020;10:71-78

DOI: $10.1159 / 000507845$

Published online: July 13, 2020

(c) 2020 The Author(s)

Published by S. Karger AG, Basel

www.karger.com/cnd

This article is licensed under the Creative Commons Attribution-NonCommercial 4.0 International License (CC BY-NC) (http://www.karger.com/Services/OpenAccessLicense). Usage and distribution for commercial purposes requires written permission.

\title{
A Family with Gitelman Syndrome with Asymptomatic Phenotypes while Carrying Reported SLC12A3 Mutations
}

\author{
Moena Ishikawa ${ }^{a}$ Yumi Tada ${ }^{b}$ Hiromu Tanaka ${ }^{a}$ Wataru Morii ${ }^{a}$ \\ Masako Inaba ${ }^{c}$ Hidetoshi Takada ${ }^{c}$ Takayasu Mori ${ }^{d}$ Emiko Noguchia \\ aDepartment of Medical Genetics, Faculty of Medicine, University of Tsukuba, \\ Tsukuba, Japan; 'bepartment of Pediatrics, Tsukuba Gakuen Hospital, Tsukuba, Japan; \\ 'Department of Child Health, Faculty of Medicine, University of Tsukuba, Tsukuba, Japan; \\ dDepartment of Nephrology, Graduate School of Medical and Dental Sciences, Tokyo \\ Medical and Dental University, Tokyo, Japan
}

\section{Keywords}

Acid-base equilibrium · Gitelman syndrome $\cdot$ Compound heterozygote

\begin{abstract}
Gitelman syndrome (GS) is an autosomal recessive disorder characterized by alkalosis, hypokalemia, and hypomagnesemia. Although hundreds of genetic variants associated with GS have been reported, many of them are categorized as of uncertain significance in ClinVar. Here, we describe a pediatric GS patient from a three-generation family whose mother and maternal grandmother were asymptomatic. The proband was a 16-year-old Japanese girl with muscle weakness and continuous hypokalemic metabolic alkalosis. The patient, her mother, and her maternal grandmother were compound heterozygous for, and each expressing a different combination of, previously reported SLC12A3 variants in GS patients. The mother and the
\end{abstract}




\section{Case Reports in Nephrology and Dialysis}

Case Rep Nephrol Dial 2020;10:71-78

DOI: 10.1159/000507845

(C) 2020 The Author(s). Published by S. Karger AG, Basel www.karger.com/cnd

Ishikawa et al.: Gitelman Syndrome Family with SLC12A3 Variants

maternal grandmother had no symptoms related to GS, and blood gas tests showed that the blood potassium levels and venous $\mathrm{pH}$ were within normal limits; however, the venous blood $\mathrm{HCO}_{3}$ - levels were slightly elevated. The phenotypic effect of missense mutations is difficult to evaluate, and accumulation of genotypic data with accurate phenotyping, including those of "healthy" and "asymptomatic" individuals in various ethnic populations, will improve the genetic diagnosis of GS.

(C) 2020 The Author(s)

Published by S. Karger AG, Basel

\section{Introduction}

Gitelman syndrome (GS) is an autosomal recessive disorder caused by pathogenic mutations of the Solute Carrier Family 12 Member 3 (SLC12A3) gene [1, 2], with a prevalence of 110 per 40,000 [1]; its symptoms are metabolic alkalosis, hypokalemia, hypomagnesemia, and elevated plasma renin and aldosterone. SLC12A3 encodes the thiazide-sensitive sodium-chloride cotransporter expressed in the renal distal convoluted tubules [3] and works to transport electrolytes to control their concentration. It is sometimes difficult to differentiate GS from other salt-losing nephropathies by using only clinical and laboratory data; therefore, genetic testing of SLC12A3 is recommended for individuals with clinically suspected GS [1]. Recently, the increasing availability of next-generation sequencing (NGS) for genetic testing allows us to find many variants in genes of interest, and the classification of variants may change with accumulation of population-based genetic data and functional studies: some variants that were previously classified as pathogenic were reclassified as benign [4], whilst others of previously unknown significance were confirmed as pathogenic. Here, we present a mutation analysis of SLC12A3 in a three-generation Japanese family including a child with GS.

\section{Case Report}

The family tree of the GS patient is shown in Figure 1a. The proband was a 16-year-old Japanese girl with muscle weakness and continuous hypokalemia and alkalosis. This condition had first been noticed when she was 10 years old. Biochemical analysis revealed hypokalemia (2.8 $\mathrm{mmol} / \mathrm{L})$; elevated plasma renin activity $(40 \mathrm{ng} / \mathrm{mL} / \mathrm{h}$ at bed rest; normal range, $0.5-2.0)$ and aldosterone $(76.5 \mathrm{ng} / \mathrm{mL}$ in the early morning at rest; normal range, 3.6-24.0); and decreased urinary excretion of calcium $(0.009 \mathrm{~g} /$ day; normal range, $0.1-0.3)$. The blood pressure was not elevated (93/48 mm Hg). Renal ultrasound revealed normal kidneys, and the blood urea nitrogen (BUN) and creatinine values were within normal range $(11.9$ and $0.56 \mathrm{mg} / \mathrm{dL}$, respectively). On the basis of the clinical symptoms and laboratory results, GS was suspected. To determine the causative mutations, NGS screening was carried out by using SPEEDI-KID [5] on the patient and her family members - her asymptomatic mother (aged 41 years) and asymptomatic maternal grandmother (aged 72 years) - and subsequently validated by Sanger sequencing using the primer pairs 5' -GCTTCACCATCAGCACACTG-3' and $5^{\prime}$-GAGGAAGGAGTGCAGGTCAG-3' for rs371443644 (NM_000339.3:c.179C>T, NP_000330.3:p.Thr60Met), 5'GCAGCTCTGGCCTAGAAAGA-3' and 5'-GCACCTGGCTTCTTGTAGATG-3' for rs79351185 (NM_000339.3:c.1706C>T, NP_000330.3:p.Ala569Val) and rs139329616 (NM_000339.3: 


\section{Case Reports in Nephrology and Dialysis}

Case Rep Nephrol Dial 2020;10:71-78

DOI: 10.1159/000507845

(c) 2020 The Author(s). Published by S. Karger AG, Basel www.karger.com/cnd

Ishikawa et al.: Gitelman Syndrome Family with SLC12A3 Variants

c.1732G>A, NP_000330.3:p.Val578Met), and 5'-CTCACССTCCTCATTCCCTA-3' and $5^{\prime}-$ GACAATCTCAGTGCCCACCT-3' for rs185927948 (NM_000339.3:c.2573T>A, NP_000330.3: p.Leu858His). In haplotype analysis for rs79351185 (p.Ala569Val) and rs139329616 (p.Val578Met), TA cloning of the PCR product was performed with the grandmother's DNA using the primers $5^{\prime}$-GCAGCTCTGGCCTAGAAAGA-3' and 5'-GCACCTGGCTTCTTGTAGATG- ${ }^{\prime}$. The PCR product was cloned into T-vector pMD20 using a Mighty TA-Cloning Kit (TaKaRa Bio Inc., Shiga, Japan) according to the manufacturer's instructions. Colonies were subjected to PCR amplification, and direct sequencing using the PCR product was performed. This study was approved by the institutional review boards of the University of Tsukuba (H30-57) and Tokyo Medical and Dental University (G2000-80), and written informed consent for publication was obtained from all the participants.

Four single-nucleotide variants in SLC12A3 that have been previously reported in GS patients were detected: rs371443644, rs79351185, rs139329616, and rs185927948 (Fig. 1b). TA cloning of the PCR products revealed that the Val allele of p.Ala569Val and the Met allele of p.Val578Met were located on different chromosomes; thus, the patient's maternal grandmother was compound heterozygous for the two variants p.Ala569Val and p.Val578Met (Fig. 2). Her asymptomatic mother carried the Met allele of p.Val578Met, so we considered that the mother inherited the Met allele of p.Val578Met from her maternal side and the His allele of p.Leu858His from her paternal side, therefore making her compound heterozygous for the two variants p.Val578Met and p.Leu858His. Because the patient's mother did not possess the Met allele of p.Thr60Met, we considered that the patient was almost certainly compound heterozygous for p.Thr60Met and p.Leu858His. The patient father's DNA was unavailable and therefore could not be genotyped.

The mother and maternal grandmother had no symptoms related to GS, and neither hypertension nor other abnormal findings suspicious for GS were pointed out in a regular medical checkup. Blood gas analysis showed that the blood potassium levels and venous $\mathrm{pH}$ were within normal limits ( 4.0 and $4.2 \mathrm{mmol} / \mathrm{L}$ for blood potassium and 7.407 and 7.358 for venous $\mathrm{pH}$, respectively; Table 1); however, the venous blood $\mathrm{HCO}_{3}$ - levels were elevated (29.6 and $28.9 \mathrm{mmol} / \mathrm{L}$, respectively; Table 1).

\section{Discussion}

GS is an autosomal recessive disorder, and genetic testing of SLC12A3 is useful to differentiate GS from other salt-losing nephropathies.

p.Thr60Met was categorized in ClinVar as a pathogenic variant and p.Leu858His as having conflicting interpretations (pathogenic and uncertain significance) [6]. Both a functional study using a mammalian cell expression system and a familial study with GS supported the pathogenicity of p.Leu858His [7, 8], and the genotyping results of the GS family in the present study also support p.Leu858His as a pathogenic variant for GS.

p.Val578Met was reported to have an allele frequency of $0.62 \%$ in the Japanese general population [9] and was categorized as of unknown significance in ClinVar [6]. Previously, a patient with GS was reported who possessed the same genotype as the mother of the present case [10]. Although the mother showed features against a diagnosis of GS (i.e., absence of hypokalemia without renal failure) [1] and did not have GS-related symptoms such as muscle 


\section{Case Reports in Nephrology and Dialysis}

Case Rep Nephrol Dial 2020;10:71-78

DOI: 10.1159/000507845

Ishikawa et al.: Gitelman Syndrome Family with SLC12A3 Variants

cramps and weakness, she did show an elevated level of $\mathrm{HCO}_{3}$ - (Table 1). p.Ala569Val was reported to have an allele frequency of $0.46 \%$ in the Japanese general population [9] and is also categorized as of unknown significance in ClinVar [6]. Individuals carrying the same genotype as the grandmother of the present case have also been reported [11], but the grandmother of the present case also showed features against a diagnosis of GS, except for the level of $\mathrm{HCO}_{3}$ - (Table 1).

Fujimura et al. [11] investigated the SLC12A3 mutations, clinical symptoms, and diagnostic opportunities of 185 Japanese GS patients. The allele frequencies of 4 variants in the general Japanese population $(n=3,552)$ [9] and those in the 185 GS patients [11] are shown in Table 2. Although not directly comparable, the difference in the allele frequencies of the variants found in the current patient (p.Thr60Met and p.Leu858His) between the general Japanese population and Japanese GS patients was much greater than that found only in the grandmother and the mother (p.Ala569Val and p.Val578Met).

In the study of Fujimura et al. [11], more than half of the diagnostic opportunity for GS was a chance blood test for diagnostic purposes other than for GS. Our data indicate that some suspected pathogenic variants such as p.Ala569Val and p.Val578Met may not be sufficient to cause phenotypic changes related to GS (i.e., hypokalemia). The blood $\mathrm{HCO}_{3}$ - levels of the mother and the grandmother and the $\mathrm{pCO}_{2}$ level of the grandmother were elevated (Table 1 ). Whilst the serum potassium level is included in many routine blood tests, the blood gas levels are not. Therefore, GS is not suspected if there is no abnormality in the blood potassium levels. No respiratory symptoms or respiratory diseases had been noticed in the mother or the grandmother, and it is possible that respiratory compensation or compensation of channels in the kidney worked to adjust the blood $\mathrm{pH}$ level. Also, the possibility cannot be denied that modifier genes are involved in the onset of GS or that phenotypic changes caused by the suspected pathogenic variants would be apparent later in life.

NGS is an effective technology to detect pathogenic variants, especially in Mendelian diseases, and is now widely used for the diagnosis of diseases of unknown etiology. The accumulation of more NGS data leads to increased numbers of variants of unknown significance, and many of those variants are very rare and sometimes unique to each individual [12]. Recently, carrier frequencies of 11 recessive newborn screening diseases were estimated using Japanese whole genome reference panels, and the actual carrier frequencies of some diseases were found to depart from the calculated carrier frequencies based on the disease incidence rates [13]. This departure may be due to inaccurate estimation of the functional effect of variants or to inaccurate diagnosis [13]. Accumulation of genotype data with accurate phenotyping, including those of "healthy" or "asymptomatic" individuals in various ethnic populations, will improve the genetic diagnosis for GS patients.

\section{Acknowledgement}

We thank all the members of the GS family who participated in this study. We are also grateful to Flaminia Miyamasu for comments that greatly improved the manuscript. 


\section{Case Reports in Nephrology and Dialysis}

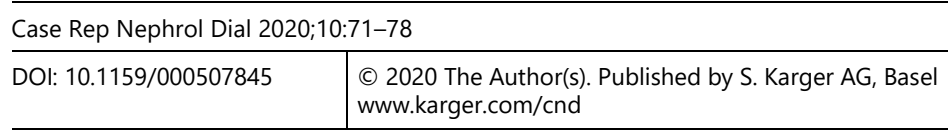

Ishikawa et al.: Gitelman Syndrome Family with SLC12A3 Variants

\section{Statement of Ethics}

This study was approved by the institutional review boards of the University of Tsukuba (H30-57) and Tokyo Medical and Dental University (G2000-80), and written informed consent for publication was obtained from all the participants.

\section{Disclosure Statement}

The authors have no conflicts of interest to declare.

\section{Funding Sources}

This work was supported by a Grant-in-Aid for Research Activity start-up from the Japan Society for the Promotion of Science.

\section{Author Contributions}

M. Ishikawa and H. Tanaka performed the experiments and drafted the manuscript. Y. Tada designed the study and collected the clinical data. W. Morii performed the data analysis. M. Inaba and H. Takada critically reviewed and revised the manuscript. T. Mori performed the experiments and critically reviewed and revised the manuscript. E. Noguchi designed the study and revised the manuscript. All the authors approved the final manuscript.

\section{References}

1 Blanchard A, Bockenhauer D, Bolignano D, Calò LA, Cosyns E, Devuyst O, et al. Gitelman syndrome: consensus and guidance from a Kidney Disease: Improving Global Outcomes (KDIGO) Controversies Conference. Kidney Int. 2017 Jan;91(1):24-33.

2 Gitelman HJ, Graham JB, Welt LG. A new familial disorder characterized by hypokalemia and hypomagnesemia. Trans Assoc Am Physicians. 1966;79:221-35.

3 Shaer AJ. Inherited primary renal tubular hypokalemic alkalosis: a review of Gitelman and Bartter syndromes. Am J Med Sci. 2001 Dec;322(6):316-32.

4 Manrai AK, Funke BH, Rehm HL, Olesen MS, Maron BA, Szolovits P, et al. Genetic Misdiagnoses and the Potential for Health Disparities. N Engl J Med. 2016 Aug;375(7):655-65.

5 Mori T, Hosomichi K, Chiga M, Mandai S, Nakaoka H, Sohara E, et al. Comprehensive genetic testing approach for major inherited kidney diseases, using next-generation sequencing with a custom panel. Clin Exp Nephrol. 2017 Feb;21(1):63-75.

6 Landrum MJ, Lee JM, Riley GR, Jang W, Rubinstein WS, Church DM, et al. ClinVar: public archive of relationships among sequence variation and human phenotype. Nucleic Acids Res. 2014 Jan;42(Database issue):D980-5.

7 Naraba H, Kokubo Y, Tomoike H, Iwai N. Functional confirmation of Gitelman's syndrome mutations in Japanese. Hypertens Res. 2005 Oct;28(10):805-9.

8 Maki N, Komatsuda A, Wakui H, Ohtani H, Kigawa A, Aiba N, et al. Four novel mutations in the thiazidesensitive $\mathrm{Na}$-Cl co-transporter gene in Japanese patients with Gitelman's syndrome. Nephrol Dial Transplant. 2004 Jul;19(7):1761-6. 


\section{Case Reports in Nephrology and Dialysis}

\begin{tabular}{l|l}
\hline Case Rep Nephrol Dial 2020;10:71-78 \\
\hline DOI: 10.1159/000507845 & $\begin{array}{l}\text { (c) 2020 The Author(s). Published by S. Karger AG, Basel } \\
\text { www.karger.com/cnd }\end{array}$
\end{tabular}

Ishikawa et al.: Gitelman Syndrome Family with SLC12A3 Variants

9 Yamaguchi-Kabata Y, Nariai N, Kawai Y, Sato Y, Kojima K, Tateno M, et al. iJGVD: an integrative Japanese genome variation database based on whole-genome sequencing. Hum Genome Var. 2015 Nov;2(1):15050.

10 Kumagai H, Matsumoto S, Nozu K. Hypokalemic rhabdomyolysis in a child with Gitelman's syndrome. Pediatr Nephrol. 2010 May;25(5):953-5.

11 Fujimura J, Nozu K, Yamamura T, Minamikawa S, Nakanishi K, Horinouchi T, et al. Clinical and Genetic Characteristics in Patients With Gitelman Syndrome. Kidney Int Rep. 2018 Sep;4(1):119-25.

12 Hoffman-Andrews L. The known unknown: the challenges of genetic variants of uncertain significance in clinical practice. J Law Biosci. 2018 Jan;4(3):648-57.

13 Yamaguchi-Kabata Y, Yasuda J, Uruno A, Shimokawa K, Koshiba S, Suzuki Y, et al.; Tohoku Medical Megabank Project Study Group. Estimating carrier frequencies of newborn screening disorders using a whole-genome reference panel of 3552 Japanese individuals. Hum Genet. 2019 Apr;138(4):389-409.

14 Theodore AC. Venous blood gases and other alternatives to arterial blood gases. In: UpToDate. Manaker S, Finlay G, editors. Waltham: Wolters Kluwer. Available from: https://www.uptodate.com [accessed 2019 July 20].

Moena Ishikawa and Yumi Tada contributed equally to this work

I

II

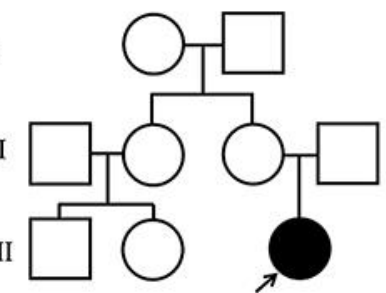

$\mathrm{I}-1$

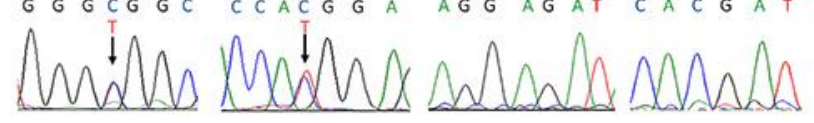

GGGCGGC CCACG G A AGG AG AT CAC G A T

II-3

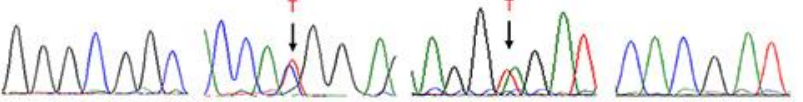

GGGCGGC CCACG GAAGGAGAT CACGAT

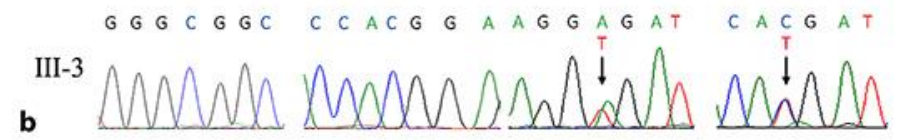

Fig. 1. a Family tree of our pediatric patient with Gitelman syndrome. b DNA sequence chromatograms of the grandmother (I-1, top), heterozygous for p.Ala569Val (rs79351185) and p.Val578Met (rs139329616); the mother (II-3, middle), heterozygous for p.Val578Met (rs139329616) and p.Leu858His (rs185927948); and the patient (III-3, bottom), heterozygous for p.Thr60Met (rs371443644) and p.Leu858His (rs185927948). 
Case Reports in Nephrology and Dialysis
Case Rep Nephrol Dial 2020;10:71-78

(c) 2020 The Author(s). Published by S. Karger AG, Basel www.karger.com/cnd

Ishikawa et al.: Gitelman Syndrome Family with SLC12A3 Variants
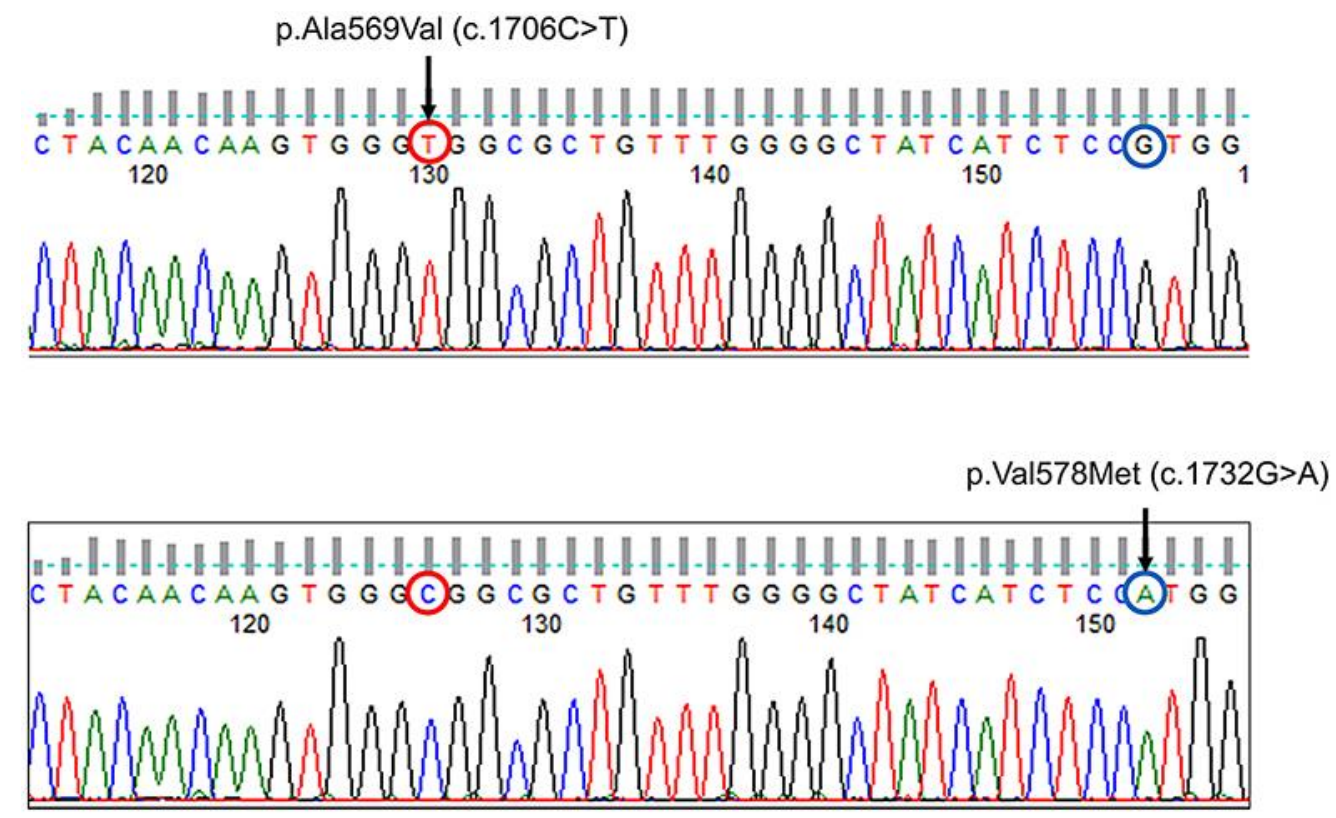

Fig. 2. TA cloning of PCR products and sequence chromatograms using DNA of the grandmother (I-1, in Fig. 1a), heterozygous for p.Ala569Val (rs79351185) and p.Val578Met (rs139329616). The red and blue circles indicate the positions of the p.Ala569Val (c.1706C >T) and p.Val578Met (c.1732G >A) variant sites, respectively.

Table 1. Clinical laboratory data of the family with Gitelman syndrome

\begin{tabular}{|c|c|c|c|c|c|}
\hline Test & Patient & Mother & Grandmother & Ref. range & \\
\hline Blood gas (vein) & & & & Ref. range (artery) & $\begin{array}{l}\text { Comparison of venous blood } \\
\text { gases to arterial values (ap- } \\
\text { proximately) }\end{array}$ \\
\hline $\mathrm{pH}$ & 7.463 & 7.407 & 7.358 & $7.35-7.45$ & $0.03-0.04 \mathrm{pH}$ units lower \\
\hline $\mathrm{pCO}_{2}, \mathrm{~mm} \mathrm{Hg}$ & 41.8 & 48.0 & 52.8 & $35-45$ & 3-8 mm Hg higher \\
\hline $\mathrm{HCO}_{3-}, \mathrm{mmol} / \mathrm{L}$ & 29.5 & 29.6 & 28.9 & $22-26$ & 1-2 mEq/L higher \\
\hline \multicolumn{6}{|l|}{ Blood electrolytes } \\
\hline $\mathrm{Na}^{+}, \mathrm{mmol} / \mathrm{L}$ & 133 & 142 & 142 & 139-146 & \\
\hline $\mathrm{Cl}^{-}, \mathrm{mmol} / \mathrm{L}$ & 92 & 106 & 106 & $96-108$ & \\
\hline $\mathrm{K}^{+}, \mathrm{mmol} / \mathrm{L}$ & 2.8 & 4.0 & 4.2 & $3.5-5.0$ & \\
\hline $\mathrm{Ca}^{++}, \mathrm{mmol} / \mathrm{L}$ & 1.08 & 1.30 & 1.30 & $1.15-1.30$ & \\
\hline
\end{tabular}

a Theodore [14]. 
Case Reports in Nephrology and Dialysis
Case Rep Nephrol Dial 2020;10:71-78

(C) 2020 The Author(s). Published by S. Karger AG, Basel www.karger.com/cnd

Ishikawa et al.: Gitelman Syndrome Family with SLC12A3 Variants

Table 2. Allele frequency of the SLC12A3 variants found in the family with Gitelman syndrome

\begin{tabular}{lll}
\hline & $\begin{array}{l}\text { Japanese, } \% \\
(n=7,104 \mathrm{a})\end{array}$ & $\begin{array}{l}\text { Japanese GS patients, \% } \\
\left(n=370^{\mathrm{b}}\right)\end{array}$ \\
\hline rs371443644 (p.Thy60Met) & 0.03 & 2.97 \\
rs79351185 (p.Ala569Val) & 0.46 & 0.81 \\
rs139329616 (p.Val578Met) & 0.62 & 1.08 \\
rs185927948 (p.Leu858His) & 1.24 & 24.3 \\
\hline
\end{tabular}

a The allele frequencies of the variants in the Japanese population were from those of the Japanese Multi Omics Reference Panel (3.5KJPNv2), Tohoku University (https://jmorp.megabank.tohoku.ac.jp) [13]. b The allele frequencies of the variants in the Japanese GS patients were from the study by Fujimura et al. [11]. 